\title{
DO PARIZA IN NAZAJ: VLADIMIR LEVSTIK IN FRANCOSKA KNJIŽEVNOST
}

Florence GACOIN-MARKS (2017). Do Pariza in nazaj: Vladimir Levstik in francoska književnost. Ljubljana: Znanstvenoraziskovalni inštitut Filozofske fakultete. ISBN 978961-237-958-2, 273 strani, 22,90€.

Florence Gacoin-Marks se donne dans son étude Un aller-retour pour Paris - Vladimir Levstik et la littérature française (le livre, rédigé en slovène dont le titre est Do Pariza in nazaj : Vladimir Levstik in francoska književnost) pour objectif de cerner les contacts littéraires de l'écrivain, traducteur, essayiste Vladimir Levstik (1886 - 1957) avec l'ensemble des aspects propres de ce qu'on appelle la civilisation française, à cette époque une civilisation mondiale. La monographie est répartie en trois chapitres qui présentent des unités autonomes : celles-ci sont pourtant organisées de façon à former une entité homogène dont l'ensemble ne pourrait exister si ses éléments organisateurs, les socles fondamentaux de l'ouvrage, n'étaient pas là.

Préalablement, Florence Gacoin-Marks met en relief le fait qu'aux yeux de Vladimir Levstik étudier le français était un signe de révolte contre l'ambiance délétère qui caractérisait « la prison des nations », i. e. la monarchie de Habsbourg (depuis 1867 le Royaume austro-hongrois). Par la lingua franca le règne de Vienne tympanisait les peuples dont les langues furent autres que l'allemand. La révolte devint la pierre angulaire sur laquelle Levstik assit son pensum vital. Le motif pour chercher à acquérir la connaissance du français résidait donc en un zélotisme panslaviste exacerbé notamment par la prépondérance des Allemands dans sa région natale, à Celje (Cilli) et dans la Carniole (Slovénie) entière. Les Slovènes furent en vérité acculés : toutes les sources en témoignent : «En tant qu'héritiers du Saint empire romain, les Germains purent asservir les Slovènes plus facilement du point de vue national et religieux. Dans la monarchie habsbourgeoise, les Slovènes furent séparés par des frontières provinciales, nationalement tout à fait contre nature. Ainsi les dirigeants étrangers (même les savants allemands) parlaient des Slovènes comme d'une race non slave ou comme d'Allemands slavisés ». ${ }^{1}$ Tout ce qui a été « germanique » répugnait Vladimir Levstik.

1 Slokan, France : Panorama littéraire slovène. Ljubljana : DSP, 1972, 15. 
Ce qui est pire : il s'agissait d'un véritable syndrome dans le sens étymologique du mot : le despotisme politique, aux yeux du jeune intellectuel, coïncidait avec le despotisme familial qu'exerçait sur le jeune styrien Mihael Levstik, son père. Celui-ci le chassa même de la maison natale, en raison de la non-conformité de l'adolescent aux normes inébranlables de l'éducation et de l'enseignement à cette époque. On dirait que pour le jeune « insurgé » au début de sa carrière il n'y eut qu'un point à l'horizon : «Le siège du monde éclairé qui se trouve aux bords de la Seine: Paris, à propos duquel le roi François I dit: "Paris, ce n'est pas une ville, c'est un monde " (cité d'après Gacoin-Marks, 2017, 11). L'initiation à la culture française devrait donc être comprise comme un pied de nez, un affront à l>autorité présentée par la Monarchie, une atteinte qui est envoyée de face et dont les séquelles seront de longue durée. ${ }^{3}$

Florence Gacoin-Marks recherche en premier lieu la façon dont Vladimir Levstik abordait l'apprentissage du français et comment. Elle découvre que les premiers pas ont été faits grâce à la célèbre méthode de Charles Toussaint et de Gustav Langenscheidt, surnommée à l'époque " Metoula » en raison de sa popularité. L'auteure met en relief le fait que Vladimir Levstik fut surtout un autodidacte qui ne manquait pas de talent. La Metoula lui permit d'acquérir les connaissances solides qui lui permirent bientôt de faire les premiers séjours dans la Capitale et de passer à ses premières adaptations des œuvres littéraires françaises. L'auteure analyse successivement Le Tartuffe, ${ }^{4}$ Le Mari de Simone, ${ }^{5}$ La Débâcle ${ }^{6}$ et Boubouroche. ${ }^{7}$ Bien que ses traductions (adaptations) ne présentent pas la fine fleur de son œuvre de traducteur postérieure (à l'exception possible de La Débâcle) elles fournirent à Levstik une expérience incontournable pour ce qu'il allait entreprendre par la suite.

Le chemin de recherche de Florence Gacoin-Marks bifurque à cet endroit pour un moment afin d'introduire le personnage qui, toute la vie durant, fut un « alter ego » de Vladimir Levstik, André Algoud (1892 - 1983). Ce partisan des idées de Charles Maurras (dans la période d'avant-guerre) et un adhérent du gaullisme (après la guerre) l'initia davantage à la culture française étant devenu son ami intime. Dans le poème - publié à titre posthume - qu'il dédia à sa famille, Vladimir Levstik est évoqué par les mots qui marquent une bienveillance profonde : «Leoustik, mon ami, mon frère des beaux jours $»^{8}$ (cité d'après Gacoin-Marks, 2017, 45).

L'influence de la famille Algoud fut double. Sur le plan idéologique elle touchait les idées de Maurras qui coïncidaient avec le monarchisme, une manière de se comporter

2 Levstik, Vladimir : Pariška legenda. Ljubljana : NUK, rokopisna zbirka, Ms 954.

3 C'est pour la même raison que Vladimir Levstik se lança dans l'étude du russe.

4 Molière, Jean-Baptiste : «Le Tartuffe ». In : CEuvres complètes. Paris : Gallimard, 1956, 681. 1.

5 Champol, Marie-Anne Bertille : Le Mari de Simone. Paris : Plon, 1896.

6 Zola, Emile : La Débacle. Paris : Gallimard, 1967.

7 Courteline, Georges : Boubouroche. Paris : Garnier, 1965.

8 Algoud, Albert André : Sur le Seuil. Paris : Éditions de Chiré, 1996, 31. 
politiquement très correcte au temps du Royaume Yougoslave et du roi Alexandre Karadjordjevich. Cela allait de pair avec la marotte qui ne cessa jamais de hanter Levstik : la haine envers les Allemands qui s'aggrava pendant la seconde guerre mondiale lorsqu'il devint victime de la SS. Il y va sans dire que les idées maurrassiennes stimulèrent le nationalisme yougoslave, qui d'ailleurs se réclamait volontiers d'être un parallèle au nationalisme français (de l'extrême droite). Ainsi Orjuna, organisation ouvrière militante à laquelle Levstik participait fut - en ce qui concerne quelques-uns de ses postulats nodaux (anticommunisme, syndicalisme jaune, corporatisme, patronat) - une sorte d'analogie à l'Action française.

L'autre ascendant qu'André Algoud exerçait sur Vladimir Levstik fut de nature littéraire. Les deux eurent une prédilection commune pour la même famille d'esprit. « Il est difficile de constater si les convictions littéraires de deux amis étaient proches par hasard ou si elles se sont formées par l'influence que l'un exerçait sur l'autre » (Gacoin-Marks, 2017, 50). Ainsi Vladimir Levstik eut un penchant pour les écrivains « qui communiquent la satire des mours sociales et politiques soit en forme des œuvres comiques (vérifier la formulation de la citation) (Molière et Gogol') soit dans les romans réalistes (Balzac, Flaubert, Zola). C'est pour cette raison que son œuvre est marquée par la satire, au plus profond »(Gacoin-Marks, 2017, 51). Les auteurs préférés de Levstik restèrent - avec ceux évoqués supra - Henri Bosco, Romain Rolland, Paul Claudel, Georges Bernanos ...

Florence Gacoin-Marks conclut la première approche de son étude par la constatation suivante : «Dans la période de son apprentissage de la langue, culture et littérature françaises Vladimir Levstik s'est formé comme traducteur habile de la littérature française. Ce qu'il prouvera entre 1913 et 1914 lorsqu'il entreprendra la traduction intégrale de Madame Bovary qui sera publiée pour la première fois en 1915. Jusqu'en 1998 cela restera la seule traduction slovène du chef-d'œuvre de Flaubert » (Gacoin-Marks, 2017, 52).

Le chapitre premier est l'anabasis du deuxième et du troisième chapitre : l'auteure y entreprend la mise en révision de grands romans français du $19^{\mathrm{e}}$ siècle tels qu'ils ont été traduits par Vladimir Levstik. Il s'agit des œuvres capitales, particulièrement accomplies qui sont les suivantes : Madame Bovary, ${ }^{9}$ La Légende et les aventures héroïques, joyeuses et glorieuses d'Ulenspiegel et de Lamme Goedzak au Pays de Flandres et ailleurs, ${ }^{10}$ Illusions perdues, ${ }^{11}$ Splendeurs et misères des courtisanes ${ }^{12}$ et Les Paysans ${ }^{13}$ et finalement Bel-Ami. ${ }^{14}$

L'analyse de la première traduction de Madame Bovary de Gustave Flaubert fait preuve de la rigueur minutieuse apportée à un travail d'érudition approfondi. En fait,

9 Flaubert, Gustave : Madame Bovary. Paris: Gallimard, 1998.

10 Coster, Charles : La Légende d'Ulenspiegel au Pays de Flandres et ailleurs. Paris : La Différence, 2003.

11 Balzac, Honoré de : « Illusions perdus «. In : La Comédie humaine. Paris : Gallimard, 1977. 5.

12 Balzac, Honoré de : «Splendeurs et misères des courtisanes «. In : La Comédie humaine. Paris : Gallimard, 1977. 6 .

13 Balzac, Honoré de : «Les Paysans ». In : La Comédie humaine. Paris : éd. Folio (publié pour la première fois à titre posthume), 1855 .

14 Maupassant, Guy de : Bel-Ami. Paris : Gallimard, 1987. 
Florence Gacoin-Marks poursuit son approche en l'appliquant d'une même discipline aux autres romans, cités ci-dessus.

Le troisième chapitre est consacré aux enjeux des ascendants littéraires à l'intérieur de l'œuvre de Vladimir Levstik. Dans son roman La Comète bleue ${ }^{15}$ Levstik reprend les procédés du roman réaliste français et russe afin de mieux mettre en relief la critique de la société slovène du début du $20^{\mathrm{e}}$ siècle. Florence Gacoin-Marks remarque que la structure du roman rappelle La Comédie humaine de Balzac. ${ }^{16}$ Il y va de même pour Hilarij Per$n a t^{17}$ qui paraît être inspiré par Le Cousin Pons. ${ }^{18}$

La monographie Paris, Un aller-retour pour Paris - Vladimir Levstik et la littérature française est le fruit du courage et de l'intuition. Florence Gacoin-Marks s'est consacrée à un auteur dont l'œuvre jusqu'à présent n'a pas été l'objet d'une étude intégrale. Elle l'a menée à bien dans une langue qui n'est pas la sienne. Néanmoins : le slovène dans lequel le livre est rédigé est académique, nec plus ultra. En plus : le livre fait témoignage de l'unité méthodologique où, dans le sens épistémologique, cartésien du mot, la décomposition du phénomène en vue d'identifier ses éléments constitutifs va de pair avec la synthèse, les deux formant une mise en évidence plus qu'efficace du sujet ciblé par ce travail. Il faut notamment savoir que les relations franco-slovènes, en ce qui concerne le domaine des contacts culturels, n'ont été exploités que trois fois, l'étude en question étant le quatrième livre sur ce sujet et le premier livre sur l'auteur éponyme.

15 Levstik, Vladimir : Višnjeva repatica. Ljubljana : Mladinska knjiga, 1983.

16 Balzac, Honoré de : La Comédie humaine. Paris: Gallimard, 1950.

17 Levstik, Vladimir : Hilarij Pernat. Maribor : Obzorja, 1995.

18 Balzac, Honoré de : » Le Cousin Pons «. In La Comédie Humaine. Paris: Gallimard, 1950. 6. 